编者按 中国医学科学院 $\boldsymbol{\&}$ 北京协和医学院血液病医院(血液学研究所)专辑

\title{
中国医学科学院\&北京协和医学院血液病医院 (血液学研究所)专辑简介
}

中国医学科学院\&北京协和医学院血液病医院(血液学研究所)(以下简称“血研所”)1957 年由中国血液学创 始人邓家栋教授奉命组建, 是我国首个血液学专业研究机构(包括附属医院). 血研所的名称曾使用过“中国人 民解放军军事医学科学院输血及血液学研究所”、“中国医学科学院输血及血液学研究所”、“中国医学科学院 血液学研究所”等. 血研所一直是我国最大的集医疗、科研、教学、产业于一体的国家级科研型血液病专业医 疗机构, 现有职工 1197 人, 在血液学及相关领域的基础研究、血液病防治、新药研制、人才培养等方面取得 了许多突出的成就. 自首次评选起, 连续 8 年获得 “中国医院专科声誉排行榜”血液学第一名, 连续 3 年获得 “中国医院科技影响力排行榜”血液病学第一名, 引领着我国血液学研究事业的发展.

成立 60 年来，血研所人一直秉承着“严谨、博精、创新、奉献”的协和精神，攻坚克难，取得累累硕果. 最 早研制出右旋糖酎代血浆, 塑料输血、输液袋, 建立新中国第一个血站和高原血库, 统一了血细胞命名, 在国 际上最早发现 AML-M2b 白血病亚型，建立了纯种近交系小鼠 615 实验白血病模型，最早在国内开展骨髓增生 异常综合征(MDS)研究, 从中药中提取出治疗慢性粒细胞白血病的新药靛玉红, 最早提出再生障碍性贫血急 慢性分型，主持全国白血病和再生障碍性贫血流行病学调查，成功进行国内第一例自体造血干细胞移植并在 全国推广. 牵头成立中华医学会血液学分会, 创立《中华血液学杂志》. 自 1960 年起开展各类培训班, 被誉为 “红色黄埔”. 伴随着新世纪的钟声, 血研所也实现了跨越式发展, 先后牵头承担多项国家重大项目, 取得了一 批具有原始创新和自主知识产权的重要科研成果，曾获得 23 个国家奖，其中与解放军第 307 医院联合申请的 “成体干细胞救治放射损伤新技术的建立与应用”获得 2014 年度国家科学技术进步奖一等奖; 具有独立知识产 权的 CAR-T 技术实现成功转让和临床应用，多项顶尖成果发表于 Nature 和 Nat Genet 等国际杂志.

\section{主要研究领域与方向}

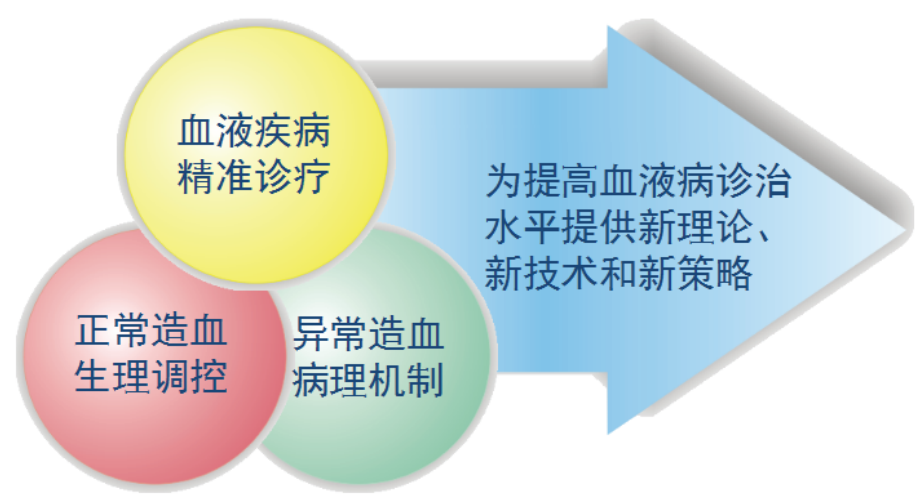


作为国内唯一的血液病三级甲等专科医院，血研所设有贫血诊疗中心、白血病诊疗中心、干细胞移植中 心、淋巴瘤诊疗中心、儿童血液病诊疗中心、MDS 诊疗中心、血栓止血诊疗中心、血液病急救中心、综合诊 疗中心、血液病专家会诊中心、血液病理和临床检测中心, 是国内血液病诊断和治疗的权威机构. 依托于血研 所的实验血液学国家重点实验室建于 1988 年, 1991 年通过国家验收后正式运转. 实验室主要从事血液学领域 特别是血液病相关的基础与转化研究, 并向国内外开放. 经过近 30 年的发展, 实验室基础设施不断完善, 逐 步形成了一支基础与临床紧密结合的高水平研究队伍. 现有固定人员 70 名, 其中法国国家医学科学院和国家 技术科学院两院院士 1 名、法国国家荣誉军团勋章获得者 1 名、国家“千人计划”特聘专家 1 名、国家“青年千 人计划”人才 2 名、国家杰出青年科学基金获得者 3 名、教育部长江学者 2 人、卫生部有突出贡献中青年专 家 7 名、国家级百千万人才 4 人、全国优秀科技工作者 2 名、天津市千人计划专家 5 名、天津市高层次创新 型领军人才 1 人. 此外, 获得国家自然科学基金创新研究群体 1 个、科技部重点领域创新团队 1 个、教育部创 新团队 1 个和协和创新团队 4 个. 重点实验室面向国家重大战略需求, 已成为我国血液学研究领域具有国内引 领、国际一流水平的集理论研究、技术开发、人才培养和学术交流为一体的开放型国家级基地. 血研所也是 国家干细胞工程技术研究中心的依托单位，是国家干细胞临床研究基地、科技部创新人才培养示范基地、教 育部内科学(血液病)、药物药理学、细胞分子生物学的全国重点学科点以及北京协和医学院干细胞与再生医学 系的发起单位. 作为北京协和医学院的下属二级院所, 培养了一批“高、精、尖”硕、博士研究生, 目前设有 7 个硕士点、 6 个博士点、 1 个博士后流动站, 并承担研究生临床教学及实习的任务. 自建所以来, 共计培养研 究生 752 名, 其中博士生 370 名, 硕士生 382 名, 每年平均为约 300 名进修生提供进修训练, 共计为全国各地 (除香港、澳门、台湾地区)的医院培养了 7000 余名血液学人才，被誉为中国血液学的“黄埔军校”.

在国家深入实施创新驱动发展战略和《健康中国 2030 规划纲要》战略部署的大背景下, 血研所准确把握 国际学术研究前沿, 充分发挥实验血液学国家重点实验室研究平台以及特有的临床资源优势, 按照中国医学 科学院的统一部署, 紧紧围绕国家健康领域重大战略需求和区域发展规划, 以“健康中国”、“创新驱动”和“京 津冀协同发展”战略为蓝图，以科技创新为动力，以学科建设为重点，提出了血研所“123”科技行动规划，即( i ) 开展中国血液病流行病学研究, 绘制中国血液病地图; (ii) 实施人类血细胞分子图谱计划并由此开发新的诊 断技术; (iii) 开发新型细胞治疗技术并制定规范标准, 力争进一步在血液肿瘤等恶性疾病发病机制、精准医疗 等方面实现新的突破.

悠悠六秩风雨，漫漫征程如歌，承载着国家重大使命而诞生的血研所已经走过一甲子风雨岁月. 她从无 到有、从弱到强、从小到大的过程, 就是中国血液学发展史的缩影. 在今后我国由大国转变为强国的“新时代” 里, 血研所人将不忘初心, 牢记使命, 抓住机遇, 团结奋进, 向着国内顶尖和世界一流的血液学研究、血液病 防治、人才培养和成果转化的国家科技创新核心基地迈进!

本专辑集中展示了血研所 14 个研究组的科研成果, 以下对各研究组的负责人分别予以介绍.

程涛, 教授, 博士生导师. 现任血研所常务副所院长, 实验血液学国家重点实验室主任, 中国医学科学院 干细胞医学中心主任, 北京协和医学院干细胞与再生医学系主任. 国家重点基础研究发展计划(973 计划)和重 点研发任务首席科学家, 国家杰出青年科学基金获得者, 国家自然科学基金重大项目以及创新研究群体负责 人, 教育部和科技部创新团队带头人. 获“千人计划”国家特聘专家、“长江学者”特聘教授、“百千万人才工程” 国家级人选、“有突出贡献”中青年专家等荣誉. 主要从事血液学和干细胞生物学方面的研究工作. 曾在 Science, Nature Medicine, Nature Genetics, Nature Cell Biology 等期刊发表论文 200 余篇(SCI 140 余篇), SCI 文章平均影 响因子 8.5, 谷歌学术被引 8000 余次(SCI 5000 余次). 获授权专利 11 项. 主编和参编中文专著 3 部、英文专著 6 部. 在本专辑中, 综述了造血微环境及其在疾病中的作用的最新研究进展.

王建祥, 主任医师, 博士生导师. 现任血研所副所院长, 国家杰出青年科学基金获得者, 国务院“政府特 殊津贴专家”, 卫生部“有突出贡献”中青年专家，首批“新世纪百千万人才工程”国家级人选，现任中华医学会 血液学分会主任委员. 主要从事白血病的临床与基础研究, 探索白血病的发病机制、靶向治疗和预后危险度分 层治疗. 作为课题负责人, 先后获得国家自然科学基金重点项目、新药创制重大专项，国家自然科学基金面上 
项目 8 项, 天津市自然科学基金 4 项. 在 PNAS, Blood, Cancer Res, Leukemia 等杂志发表论文 80 余篇. 在本专 辑中, 就 CAR 的基本结构、CAR 结构的演变、在急性白血病治疗中的进展以及目前 CAR-T 设计新方案四个 方面进行叙述. 同时也详细介绍了 $\mathrm{CD} 19 \mathrm{scFv} / \mathrm{CD} 80$ 融合蛋白联合输注淋巴细胞在促使逃避免疫监视的 B-ALL 细胞有效转化成抗原提呈细胞, 进而激活特异性免疫反应中的作用.

韩忠朝, 教授, 博士生导师. 实验血液学国家重点实验室学位委员会主任. 法国医学科学院外籍院士、法 国技术科学院外籍院士、教育部“长江学者”特聘教授、国家杰出青年科学基金获得者、卫生部有突出贡献中 青年专家、全国优秀科技工作者、中国生物医学工程学会干细胞工程技术分会主任委员、国家干细胞工程技 术研究中心主任等. 获得 2001 年度国家自然科学二等奖(第一完成人)、2009 年度国家科技进步二等奖(第一完 成人)、2014 年度国家科技进步一等奖(第二完成人). 全球首家脐带间充质干细胞库建立者, 首创外周血造血 干细胞治疗下肢缺血性疾病新技术. 从事血液学、血管细胞生物学以及干细胞工程技术及其转化应用的研究, 共发表论文 400 余篇, 其中在 Lancet, Blood 等国际杂志发表 200 余篇, 被引用 5600 余次, 授权发明专利 19 项. 韩忠朝教授于 2017 年在《中国科学: 生命科学》发表了关于间充质干细胞(MSCs)的基础及临床应用的综述性 文章. 在本专辑中, 主要介绍了 MSCs 的基本生物学功能, 然后进一步分析了 MSCs 的一般临床应用范围, 并 总结了现有已批准上市的 MSCs 临床治疗产品, 为 MSCs 的产业化和研究提出了未来的方向.

郑国光, 研究员, 博士生导师. 中国生理学会血液学专业委员会委员、中华医学会医学细胞生物学分会委 员、天津市免疫学会第二届理事会常务理事、天津细胞生物学会理事. 主要研究方向为运用分子生物学、细 胞生物学和实验动物手段, 探索白血病微环境形成机制以及白血病微环境与造血干细胞、白血病干细胞相互 作用机制; 细胞因子、嘌呤受体等异常细胞间通讯在白血病发生、发展中的作用机制. 作为第一作者和通讯作 者在 Cancer Res, Oncoimmunol, J Hematol Oncol, Haematologica, Oncotarget, J Immunol, JBC, Cell Cycle, Mol Immunol, Stem Cell Rev, Tumor Biol, Leukemia Res, BBRC, Leukemia \& Lymph, Protein Expres \& Purifi 等国内、外 知名期刊发表论文 49 篇(SCI 论文 24 篇). 目前主持国家自然科学基金 2 项、中国医学科学院医学与健康科技 创新工程 2 项、天津市基础研究重点课题 1 项. 在本专辑中, 介绍了巨噬细胞在血液系统恶性疾病中的表型、 功能特点以及在疾病进展中的作用, 有助于更好地理解疾病微环境的形成和在疾病进展中的作用机制.

Hideo Ema(依马秀夫), 研究员, 干细胞与再生医学专业博士生导师. 依马秀夫教授先后在美国国立卫生 研究院(NIH)、普林斯顿大学、法国巴斯德研究所及日本筑波大学等进行了多年干细胞尤其是造血干细胞相关 研究, 自 2014 年起于血研所任全职特聘教授. 目前主要研究方向是造血干细胞的正常和异常造血及造血干细 胞的体外扩增. 依马秀夫教授在 Cell, Immunity, Blood, JEM, PNAS 等刊物上发表文章 50 多篇. 由他领导的团队 建立的造血干细胞单细胞的分选及体内移植方法发表在 Nature Protocol 上, 被引用百余次. 本专辑里的两篇 文章, 一篇是围绕单细胞移植技术的操作和应用进行介绍, 另一篇则围绕急性 B 淋巴细胞白血病的发生机制 进行综述.

袁卫平, 研究员, 博士生导师(双专业: 细胞生物学, 干细胞与再生医学), 中国医学科学院/北京协和医学 院“协和学者”特聘教授. 2009 年底回国后主要从事运用不同模式动物, 研究关键信号通路在正常造血、造血衰 老及异常造血中的作用, iPSC 技术研究及其在细胞治疗中的应用. 近年来已在多种国际重要学术期刊, 包括 Blood, Cell Res, Cell Stem Cell, Circul Res, Genome Biol, J Cell Biol, Leukemia, Nature, Nat Commun, Nat Genet, Nat Med 等期刊发表文章 70 余篇, 引用超千次. 目前主持和参加国家自然科学基金及科技部国家干细胞重大 专项等多项国家级课题/子课题. 担任国内外多个学术协会的理事、委员或会员. 在本专辑里, 详细介绍了 MLL-NRIP3 融合基因在诱发白血病发生中的作用机制.

张孝兵, 研究员, 博士生导师. 在血液学和干细胞领域有近 20 年研究经验, 其中在海外工作 15 年. 团 队的研究聚焦于临床转化医学, 主要方向为利用 CRISPR/Cas9 革命性基因编辑技术治疗血友病等单基因遗传 病, 利用 Cas $9 / \mathrm{sgRNA}$ 人全基因组慢病毒文库进行功能基因组学研究, 重编程人血细胞为 iPSC, MSC, NSC 等 干细胞, 重塑造血微环境以促进干细胞增殖等. 在 Cell Res, J Clin Invest, PLoS Med, Stem Cells, PNAS 等国际 学术杂志发表 30 多篇论文. 担任 Stem Cells 等杂志的审稿人. 目前主持国家自然科学基金项目并参加其他 
重大课题. 在本专辑中, 介绍了造血干细胞移植及基因治疗的最新进展和面临的挑战.

高瀛岱，研究员，博士生导师. “协和学者”特聘教授, “重点研发计划”课题负责人. 主要研究方向为靶向血 液干细胞的药物研究. 在 Nat Commun, Blood, Leukemia, JMC, Cancer Immunol Immunother 等期刊发表文章多 篇. 目前主持国家自然科学基金、“973”子项目各 1 项, 获得省部级奖励 4 项. 在本专辑中, 主要介绍了白血病 干细胞的主要生物学特征, 以及靶向治疗白血病的方法.

冯晓明，研究员，博士生导师. 国家“青年千人计划”，国家自然科学基金委员会“优秀青年”科学基金获得 者，“协和学者”特聘教授，天津市“131”创新型人才第一层人选，曾获美国 Wistar 研究所 Ching Jer Chern Memorial Award. 主要研究方向为肿瘤免疫和干细胞免疫; 运用条件性基因敲除小鼠模型和基因表达调控平 台, 以及疾病模型研究 $\mathrm{T}$ 淋巴细胞分化与功能的调控机制; 细胞静息和活化调控分子在抗肿瘤/白血病 $\mathrm{T}$ 细胞 免疫中的作用; 干细胞的免疫特性和免疫调节功能. 先后在 Nat Immunol, Blood, Nat Commun 等期刊发表多篇 文章. 在本专辑中, 从表观遗传学、胞内信号通路和翻译后修饰等角度对调节性 $\mathrm{T}$ 细胞的 FoxP3 表达及稳定 性的控制机制研究进行了简要概述.

杨仁池，主任医师，博士生导师．血栓止血诊疗中心主任，现为国家血友病病例信息管理中心负责人，中 华医学会血液学分会止血与血栓学组副组长, 天津市医学会内科学分会委员. 主要研究方向为免疫性血小板 减少症(ITP)的发病机制, 包括间充质干细胞诱导 ITP 患者免疫耐受、 microRNA 对于 ITP 患者的免疫调控等. 先后在 SCI 期刊上作为第一作者或通讯作者发表论文 76 篇. 先后主持国家公益卫生行业科研专项基金、国家 自然科学基金、天津市自然科学基金重点项目等. 先后获得国家科学技术进步二等奖、天津市科学技术进步 一等奖、教育部科学技术进步二等奖等多项奖励. 在本专辑中, 以地中海贫血、血小板无力症和血友病为代表, 简述了目前遗传性血液病的研究进展及尚需解决的问题.

张风奎，主任医师，博士生导师. 贫血诊疗中心主任，兼任血液病医院国家药物临床试验机构主任. 中华 血液学杂志副主编、白血病淋巴瘤杂志编委. 主要研究方向为红细胞疾病基础与临床, 从事各种骨髓造血衰竭 发病机制、诊断与鉴别诊断、预后判断及治疗等研究，对溶血性贫血、骨䯣增生异常综合征和少见疑难贫血 的诊断经验丰富. 再生障碍性贫血免疫抑制治疗疗效国内领先，率先报告我国大颗粒淋巴细胞白血病、先天性 红细胞生成异常性贫血临床特征. 发表文章多篇. 在本专辑中, 介绍了深度基因测序时代分子遗传学克隆性 造血在再生障碍性贫血检出的意义, 有助于更好理解再生障碍性贫血造血细胞生物学特征和疾病转化. 目前 主持、参与国家科技重大专项课题 2 项，天津市自然科学基金项目 1 项.

张否, 主任医师, 博士研究生导师, “协和学者”特聘教授、中国血液生理学专业委员会青年委员、中华医 学会血液学分会止血与血栓组委员、中华血液学杂志通讯编委. 主要从事血栓与止血疾病的临床与基础研究, 先后在 Leukemia, Haematologica, Exp Hematol, Hemophilia 等期刊发表文章 28 篇. 主持国家自然科学基金 3 项、 “863”子课题 1 项、科技部国际合作子课题 1 项、Bayer Hemophilia Award 1 项和天津市自然科学基金项目 2 项. 获国家级奖 1 项, 获省部级奖 4 项. 骨髓增殖性肿瘤是起源于多能造血干细胞的恶性骨髓增殖性疾病, 在 本专辑中，对该病的机制、临床表现及诊疗等方面的研究进展进行了综述.

周家喜，研究员，博士生导师，“协和学者”特聘教授，中国细胞生物学会青年理事会理事，中国细胞生物 学会干细胞分会理事、天津市细胞生物学会副理事长, 国际干细胞研究协会(ISSCR)会员. 2010 年底加入血研 所. 目前主要运用人类胚胎干细胞和 iPS 细胞以及造血干细胞作为研究模型, 利用 CRISPR/Cas 9 技术研究早期 造血分化以及向巨核细胞分化并产生血小板的机理和应用. 先后在 Cell Res, PNAS, J Cell Biol, Stem Cell Rep, Stem Cells, Stem Cells Trans Med 等杂志作为第一作者和通讯作者发表文章近 30 篇. 目前主持国家重大研发子 项目、国家自然科学基金、天津市自然科学基金重点项目、中国医学科学院创新工程等项目. 在专辑中, 报道 了综合利用脐带血来源造血祖细胞和人多能干细胞巨核分化模型，结合转录组测序和功能验证篮选调控巨核 分化和血小板产生关键基因的策略.

石莉红，博士生导师，研究员.中组部第十二批“千人计划”青年人才、“协和学者”特聘教授，天津市“131” 创新型人才培养工程第一层次人选. 研究方向主要包括诱导多能干细胞向人红细胞发育分化的转录调控机制 
研究, 和红细胞疾病的发病机制及其治疗. 已在 Nat Med, PLoS Genet, HMG, Bioinformatics, Blood, PNAS, MCB 等国际期刊上发表 SCI 文章 30 余篇, 主持并参与了多个国家、省市级科研项目. 在本专辑中, 小结了血红素 在红细胞中的合成调控、对红细胞稳态维持及血红素异常导致的红细胞疾病.
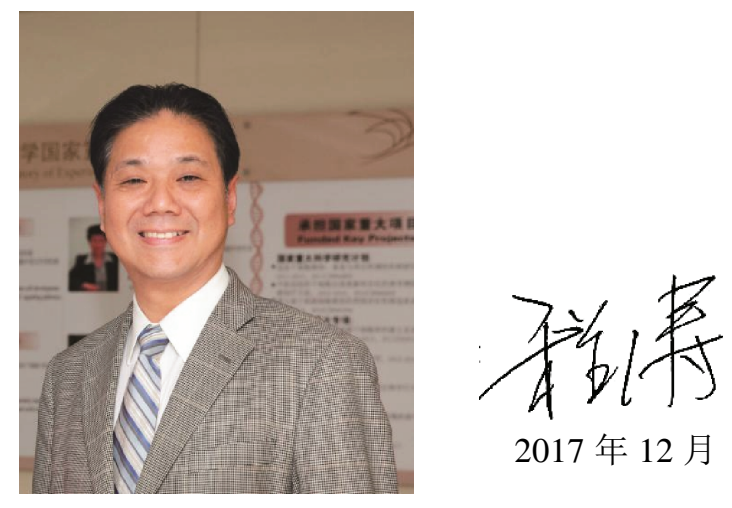

2017 年 12 月 